\section{High mortality following gastrostomy tube insertion in adult peritoneal dialysis patients: case report and literature review}

Table 1 Summary of case reports of patients on peritoneal dialysis and gastrostomy tube feeding by time elapsed between percutaneous endoscopic gastrostomy (PEG) and peritoneal dialysis (PD) catheter insertion.

\begin{tabular}{|c|c|c|c|c|c|c|}
\hline $\begin{array}{l}\text { Case } \\
\text { report }\end{array}$ & $\begin{array}{l}\text { Age } \\
\text { in } \\
\text { years }\end{array}$ & Sex & $\begin{array}{l}\text { Cause of } \\
\text { end-stage } \\
\text { renal disease }\end{array}$ & $\begin{array}{l}\text { Time elapsed } \\
\text { between PEG } \\
\text { and PD catheter } \\
\text { insertion }\end{array}$ & Complications & Outcome \\
\hline $\begin{array}{l}\text { Lew et al., } \\
2011\end{array}$ & $\mathrm{~N} / \mathrm{A}$ & M & Amyloidosis & 14 months & None & $\begin{array}{l}\text { Later death due } \\
\text { to unrelated } \\
\text { cause }\end{array}$ \\
\hline $\begin{array}{l}\text { Fein et al., } \\
2001\end{array}$ & 77 & $\mathrm{~F}$ & $\begin{array}{l}\text { Vascular dis- } \\
\text { ease }\end{array}$ & $\mathrm{N} / \mathrm{A}$ & Peritonitis $\times 2$ & $\begin{array}{l}\text { Later death due } \\
\text { to unrelated } \\
\text { cause }\end{array}$ \\
\hline $\begin{array}{l}\text { Fein et al., } \\
2001\end{array}$ & 69 & M & Diabetes & 4 years & None & $\begin{array}{l}\text { Later death due } \\
\text { to unrelated } \\
\text { cause }\end{array}$ \\
\hline
\end{tabular}

F, female; M, male; N/A, data not available.

Table 2 Summary of case reports of patients on peritoneal dialysis and gastrostomy tube feeding providing duration of withholding peritoneal dialysis (PD) after percutaneous endoscopic gastrostomy (PEG) insertion.

\begin{tabular}{|c|c|c|c|c|c|c|}
\hline $\begin{array}{l}\text { Case } \\
\text { report }\end{array}$ & $\begin{array}{l}\text { Age } \\
\text { in } \\
\text { years }\end{array}$ & Sex & $\begin{array}{l}\text { Cause of } \\
\text { end-stage } \\
\text { renal } \\
\text { disease }\end{array}$ & $\begin{array}{l}\text { Duration of } \\
\text { withholding } \\
\text { PD after PEG } \\
\text { insertion }\end{array}$ & $\begin{array}{l}\text { Complications } \\
\text { (onset from time } \\
\text { of insertion) }\end{array}$ & Outcome \\
\hline $\begin{array}{l}\text { Dahlan et al., } \\
2013 \text { (pres- } \\
\text { ent study) }\end{array}$ & 79 & $\mathrm{~F}$ & $\begin{array}{l}\text { Multiple } \\
\text { myeloma }\end{array}$ & $\begin{array}{l}\text { Switched to } \\
\text { hemodialysis }\end{array}$ & $\begin{array}{l}\text { Leak, polymicro- } \\
\text { bial peritonitis } \\
\text { ( } 8 \text { days) }\end{array}$ & $\begin{array}{l}\text { Death due to } \\
\text { peritonitis }\end{array}$ \\
\hline $\begin{array}{l}\text { Fein et al., } \\
2001\end{array}$ & 37 & M & $\begin{array}{l}\text { Obstructive } \\
\text { uropathy }\end{array}$ & 48 hours & $\begin{array}{l}\text { Leak, Klebsiella } \\
\text { peritonitis }\end{array}$ & Recovered \\
\hline $\begin{array}{l}\text { Fein et al., } \\
2001\end{array}$ & 81 & $\mathrm{~F}$ & $\begin{array}{l}\text { Diabetes } \\
\text { mellitus }\end{array}$ & 2 weeks & $\begin{array}{l}\text { Peritonitis while } \\
\text { PD on hold } \\
\text { ( } 10 \text { days) }\end{array}$ & $\begin{array}{l}\text { Later death due } \\
\text { to unrelated } \\
\text { cause }\end{array}$ \\
\hline $\begin{array}{l}\text { Fein et al., } \\
2001\end{array}$ & 44 & M & ? AIDS & $\begin{array}{l}\text { Switched to } \\
\text { hemodialysis }\end{array}$ & None & $\begin{array}{l}\text { Later death due } \\
\text { to unrelated } \\
\text { cause }\end{array}$ \\
\hline $\begin{array}{l}\text { Fein et al., } \\
2001\end{array}$ & 66 & $\mathrm{~F}$ & $\begin{array}{l}\text { Diabetes } \\
\text { mellitus }\end{array}$ & None & $\begin{array}{l}\text { Leak, fungal peri- } \\
\text { tonitis (<1 day) }\end{array}$ & $\begin{array}{l}\text { Death due to } \\
\text { peritonitis }\end{array}$ \\
\hline $\begin{array}{l}\text { Fein et al., } \\
2001\end{array}$ & 58 & M & $\begin{array}{l}\text { Diabetes } \\
\text { mellitus }\end{array}$ & $\begin{array}{l}\text { Switched to } \\
\text { hemodialysis }\end{array}$ & $\begin{array}{l}\text { Leak, polymicro- } \\
\text { bial peritonitis } \\
\text { (7 days) }\end{array}$ & $\begin{array}{l}\text { Death due to } \\
\text { peritonitis }\end{array}$ \\
\hline $\begin{array}{l}\text { Fein et al., } \\
2001\end{array}$ & 64 & $\mathrm{M}$ & $\mathrm{N} / \mathrm{A}$ & 6 weeks & $\begin{array}{l}\text { Fungal peritonitis } \\
\text { (50 days) }\end{array}$ & $\begin{array}{l}\text { Death due to } \\
\text { peritonitis }\end{array}$ \\
\hline $\begin{array}{l}\text { Fein et al., } \\
2001\end{array}$ & 86 & M & $\mathrm{N} / \mathrm{A}$ & $\begin{array}{l}\text { Switched to } \\
\text { hemodialysis }\end{array}$ & None & $\begin{array}{l}\text { Later death due } \\
\text { to unrelated } \\
\text { cause }\end{array}$ \\
\hline $\begin{array}{l}\text { Fein et al., } \\
2001\end{array}$ & 69 & $M$ & $N / A$ & $\begin{array}{l}\text { Switched to } \\
\text { hemodialysis }\end{array}$ & None & $\begin{array}{l}\text { Later death due } \\
\text { to unrelated } \\
\text { cause }\end{array}$ \\
\hline $\begin{array}{l}\text { Goel et al., } \\
1998\end{array}$ & $\mathrm{~N} / \mathrm{A}$ & $\begin{array}{l}\mathrm{N} / \\
\mathrm{A}\end{array}$ & $\mathrm{N} / \mathrm{A}$ & $\mathrm{N} / \mathrm{A}$ & Peritonitis & $\mathrm{N} / \mathrm{A}$ \\
\hline $\begin{array}{l}\text { Goel et al., } \\
1998\end{array}$ & $\mathrm{~N} / \mathrm{A}$ & $\begin{array}{l}N / \\
A\end{array}$ & $\mathrm{~N} / \mathrm{A}$ & $\mathrm{N} / \mathrm{A}$ & None & $\mathrm{N} / \mathrm{A}$ \\
\hline
\end{tabular}

F, female; M, male; N/A, data not available.
A 79-year-old woman on peritoneal dialysis was admitted to hospital on January 14, 2013, for management of bilateral nonhealing heel ulcers. During her hospital stay, her oral intake was very poor and her albumin level progressively declined to $8 \mathrm{~g} / \mathrm{L}$. On February 15, she underwent percutaneous endoscopic gastrostomy (PEG) tube insertion and was switched to hemodialysis. Prophylactic oral fluconazole and intravenous piperacillin/tazobactam were administered. On February 23, she developed fever, hypotension, and tachycardia, and subsequently had a cardiorespiratory arrest. She was resuscitated and transferred to the intensive care unit. A leak around the PEG tube site was noted and the PD catheter drained turbid fluid with a total white cell count of $9800 \times 10^{-6} / \mathrm{L}$ and effluent culture grew Klebsiella oxytoca, Pseudomonas aeruginosa, Enterococcus species, and Candida albicans. Imaging showed no evidence of bowel perforation. The patient continued to deteriorate and she died on February 24, 2013.

A very limited number of case reports [1, 2 ] indicate that the insertion of peritoneal dialysis catheters in patients who have a preexistent and presumably well-healed PEG may be safe ( Table 1 ), but that the insertion of gastrostomy tubes in patients receiving peritoneal dialysis is associated with major adverse outcomes including leaks and fatal or nonfatal peritonitis ( Table 2) [2,3]. With our patient, withholding peritoneal dialysis, switching to hemodialysis, and use of prophylactic antimicrobials did not prevent the development of fatal peritonitis. We hypothesize that residual peritoneal fluid may have prevented effective healing of the PEG site with subsequent spillage of gastric contents into the peritoneal space. It is uncertain whether surgical as opposed to endoscopic placement or a longer healing time would have resulted in a better outcome. We conclude that gastrostomy tubes should not be placed in adult patients on peritoneal dialysis, because of a very high rate of fatal peritonitis.

Endoscopy_UCTN_Code_CPL_1AH_2AI

Competing interests: None

\section{R. Dahlan, M. Biyani, B. B. McCormick}

Division of Nephrology, Department of Medicine, University of Ottawa and the Ottawa Hospital, Ottawa, Ontario, Canada 


\section{References}

1 Lew SQ Gruia A, Hakki F. Adult peritoneal dialysis patient with tenckhoff and percutaneous endoscopic gastrostomy catheters. Perit Dial Int 2011; 31: 360

2 Fein PA, Madane SJ, Jorden A et al. Outcome of percutaneous endoscopic gastrostomy feeding in patients on peritoneal dialysis. Adv Perit Dial 2001; 17: 148-152

3 Goel S, Ribby KJ, Kathuria P et al. Temporary stoppage of peritoneal dialysis when laparoscopic procedures are performed on patients undergoing CAPD/CCPD: a change in policy. Adv Perit Dial 1998; 14: 80-82
Bibliography

DoI http://dx.doi.org/

10.1055/s-0033-1344408

Endoscopy 2013; 45: E313-E314

(c) Georg Thieme Verlag KG

Stuttgart · New York

ISSN 0013-726X
Corresponding author

\section{Dr B. McCormick}

1967 Riverside Dr

Ottawa

Ontario K1H 7W9

Canada

Fax: +613-738-82893

bmccormick@ottawahospital.on.ca 